\title{
Nachweis eines Pharynxbulbus bei Polychaeten aus der Familie Flabelligeridae
}

\author{
M. WILFERT \\ Institut für Zoologie, Universität Düsseldorf; \\ Düsseldorf, Bundesrepublik Deutschland
}

\begin{abstract}
Evidence of a buccal bulb in polychaetes of the family Flabelligeridae. The fore-gut of the flabelligerid polychaetes Flabelligera diplochaitus and Stylarioides monolifer was investigated histologically. In contrast to Dales $(1962,1963)$ a buccal bulb was found. Important parts of the stomodeum in flabelligerids are a blindly-ending, separate pharynx sac (stomodeal sac) whose epithelium consists of cylindrical and vacuolized cells and is provided with a cuticle, a buccal bulb comprising muscles and vacuolized cells, a sagittal muscle system (investing muscles of the bulb), and a retractor muscle of the bulb. A comparison with the stomodeum of other polychaetes shows that detailed investigations, which might provide a basis for phylogenetic studies, are still lacking.
\end{abstract}

\section{EINLEITUNG}

Bei vielen Polychaeten findet sich unter dem ventralen Teil des Pharynxepithels ein hervorstreckbarer Bulbus (Schlundkopf, Schlundsack, buccal organ, buccal bulb, bulbe pharyngien). Zusammenfassende Darstellungen über sein Vorkommen bei den einzelnen Familien gaben Jeener (1932), Dales $(1962,1963)$ und Orrhage (1964, 1973).

DaLES (1962, 1963) verwendete das Vorhandensein oder Fehlen eines solchen Bulbus als Merkmal zur Klärung phylogenetischer Zusammenhänge einzelner Polychaetenfamilien; es bildete ein wesentliches Hilfsmittel für seine Konstruktion verschiedener Stammbäume. Dies stieß auf Kritik von STORCH (1968), vor allem aber von Orrhage $(1973,1974)$. Orrhage (1973) konnte zeigen, daß bisher viel zu wenig anatomische und histologische Untersuchungen der Schlundapparate von Polychaeten vorliegen, die ein derart weitreichendes Systematisieren der einzelnen Familien gestatten.

Der vorliegende Beitrag ist im Sinne der Forderungen ORRHAGEs nach ausreichendem Material zur Festigung oder Widerlegung von DaLes' Ansichten zu verstehen. Lichtmikroskopisch wurde der Pharynxbulbus von zwei Angehörigen der Familie Flabelligeridae untersucht; bisher fehlte eine Untersuchung des Vorderdarms von Arten dieser Familie. Die erbrachten Befunde wurden mit den sich widersprechenden Angaben in der Literatur verglichen. 


\section{MATERIAL UND METHODEN}

Untersucht wurden die Arten Flabelligera diplochaitus (Отто) und Stylarioides monolifer Delle Chiaje. $F$. diplochaitus stammte von der Insel St. Katarina bei Rovinj (Istrien, Jugoslawien), S. monolifer wurde in Morgat (Bretagne, Frankreich) gesammelt. Die Fixierung erfolgte in Bouin-Duboscq-Brasil oder Formol-Alkohol, die Einbettung in Paraffin (Schnittdicke: $5 \mu \mathrm{m}$ ). Es wurden Sagittal- und Querschnittserien durch die Vorderenden angefertigt (Färbung mit Azan oder Mallory's Trichrome).

\section{ERGEBNISSE}

\section{Bau des Vorderdarms von Flabelligeradiplocbaitus}

Die histologische Unterschung von Flabelligera diplochaitus ergab das Vorhandensein eines ventralen Pharynxbulbus. Seine wesentlichen Teile und seine Beziehungen zu weiteren Strukturen im Vorderdarmbereich sind der Abbildung 1 zu entnehmen, die einen Sagittalschnitt durch den Vorderkörper darstellt.

Von der Ventralseite des Pharynx entspringt distal von der Mundöffnung ein

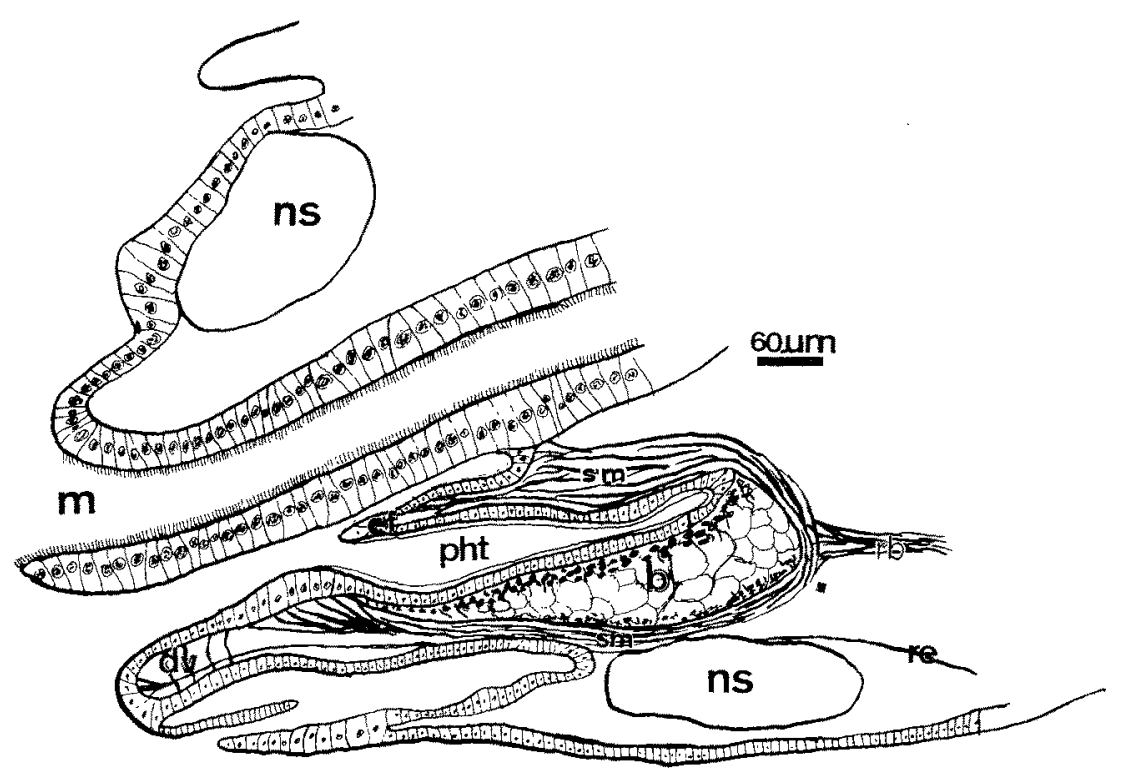

Abb. 1: Schematischer Sagittalschnitt durch das Vorderende von Flabelligera diplochaitus. Abkürzungen: $\mathrm{b}=$ Pharynxbulbus, $\mathrm{dm}=$ Darmmuskulatur, $\mathrm{dv}=$ Dorsoventralmuskulatur, ef = Falte des Pharynxtaschen-Epithels, epp = Epithel der Pharynxtasche, epv = Epithel des Vorderdarms, $\mathrm{ku}=$ Kutikula des Pharynxtaschen-Epithels, $\mathrm{m}=$ Mund, $\mathrm{mz}=$ Muskelzellen im Bulbus, $n s=$ Nervensystem, pht $=$ Pharynxtasche, $r b=$ Bulbus-Retraktor, re = Retraktor am ventralen Epithel, $\mathrm{sm}=$ Sagittalmuskel-System, $v z \mathrm{~b}=$ Blasenzellen des Pharynxbulbus, vzp $=$ Blasenzellen des Pharynxtaschen-Epithels 
Epithel, das sich unter Bildung einer paarigen, jeweils halbkreisförmig gerundeten Falte proximad erstreckt, wo es in Höhe der Mundöffnung in das ventrale Körperepithel übergeht.

Dadurch wird zwischen ventraler Pharynxwand und dem erwähnten Epithel ein Hohlraum geschaffen, der hier als Pharynxtasche (Abb. 1) bezeichnet wird. Von den Epithelfalten wird diese Tasche in zwei Stockwerke geteilt.

Pharynxtaschen- und Pharynxepithel sind histologisch deutlich unterscheidbar (Abb. 1, 2). Die eigentlichen Darmzellen bilden einen $50 \mu \mathrm{m}$ hohen Zellverband, der Cilien trägt, während der größte Teil des Pharynxtaschenepithels von nur ca. $20 \mu \mathrm{m}$ großen Zellen gebildet wird, die cilienlos sind, jedoch eine Kutikula abscheiden. Lediglich die Zellen des blindgeschlossenen Endes des unteren hinteren PharynxtaschenBezirks sind anders differenziert. Sie bestehen aus abgerundeten, optisch weitgehend leer erscheinenden Zellen, die dem Typ der „Blasenzellen“ (vgl. Welsch \& Storch, 1972) entsprechen (Abb. 2).

Dem ventralen Epithel der Pharynxtasche liegt der Pharynxbulbus an (Abb. 1, 2), der unterhalb der Epithelfalten liegt und sich bis zum Ende der Pharynxtasche er-

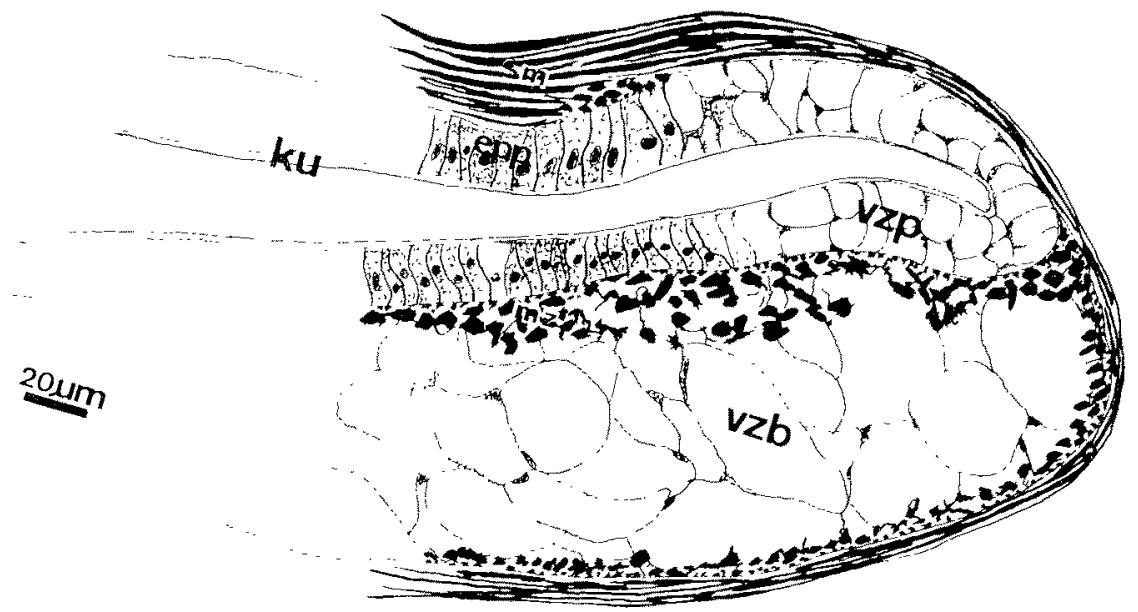

Abb. 2: Schema des blindgeschlossenen Endes der Pharynxtasche von Flabelligert diplochaitus

streckt. Der im Querschnitt annähernd nierenförmige Bulbus ist distal halbkugelig gerundet, sein proximales Ende in der Mitte leicht einwärts gebogen, so daß er lateral je einen nach vorn vorspringenden Zipfel besitzt.

Dorsal wird der Bulbus vom Pharynxtaschen-Epithel bedeckt. Ventral und lateral dagegen grenzt ihn ein Sagittalmuskelsystem (Abb. 1, 2) gegen das Cölom ab. Es erstreckt sich von der Ubergangsstelle Parynxtaschen-/ventrales Körperepithel bis in die Spitze der Epithelfalten, wobei es am distalen Bulbusende und dem blindgeschlossenen Ende der Pharynxtasche von distaler in proximaler Richtung umbiegt.

Diese Umbiegungsstelle bildet ferner die Angriffsfläche für einen Retraktor des Bulbus, der distal in den ventralen Abschnitt des Hautmuskelschlauchs ïbergeht (Abb. 1). Parallel zu diesem Muskelsystem ist unterhalb des Sagittalmuskels ein wei- 
terer Muskel zwischen vorderer ventraler Epidermis und ventralem Hautmuskelschlauch ausgespannt. Als viertes Muskelsystem treten feine Fasern hinzu, die dorsoventral zwischen Pharynxtaschen-Epithel und Epidermis verlaufen (Abb. 1). Das Zusammenwirken dieser vier Muskelgruppen beim Einziehen und Ausstülpen des Bulbus in Zusammenarbeit mit Cölomdruckänderungen dürfte in ähnlicher Weise wie bei Ctenodrilus vor sich gehen (WILFERT, 1973).

\section{Histologie des Bulbus von Flabelligera diplocbaitus}

Der Hauptteil des Bulbus besteht aus blasigen Zellen, die auf Schnitten im Lichtmikroskop nahezu leer erscheinen und weitgehend an die Blasenzellen im Pharynxtaschenepithel erinnern (Abb. 2). Sie sind jedoch vor allem wesentlich größer als diese, $z$. T. erreichen sie einen Durchmesser von $35 \mu \mathrm{m}$.

Diese Blasenzellen werden vollständig eingehüllt von Muskelzellen, die mit ihrem kontraktilen Material zirkulär unter dem Sagittalmuskel bzw. dem Pharynxtaschenepithel verlaufen. Proximales und distales Bulbusende besitzen zusätzlich transversal ausgespannte Muskelfasern, einige derartige Zellen durchqueren den Bulbus auch in dorsoventraler Richtung.

AuBere Anatomie von Bulbus und Pharynxtasche bei Stylarioides monolifer

Wie bei Flabelligera diplochaitus ergab die histologische Untersuchung das Vorhandensein eines Bulbus auch bei Stylarioides monolifer. Die gegenseitigen Lagebeziehungen von Pharynxbulbus, Pharynxtasche und Sagittalmuskelsystem sind bei dieser Art jedoch komplizierter und werden am deutlichsten bei der Betrachtung von Querschnitten (Abb. 3).

Der Vorderdarm beginnt am Mund mit einem mit Cilien versehenen Epithel, sein Lumen erscheint im Querschnitt T-förmig (Abb. 3a). Noch im Bereich der Mundöfnung wird das Darmlumen ventrad durch das Lumen einer Pharynxtasche erweitert (Abb. 3b-i). Dessen Wandung besteht aus einem wimperlosen Epithel, das in ventraler Richtung rom Darmepithel ausgeht und sich schließlich mit der ventralen Epidermis vereinigt (Abb. 3b).

Das Pharynxtaschenepithel bildet mehrere Falten, die ins Lumen der Tasche hineinragen. Die oberen paarigen Falten (Abb. 3e) entsprechen den Epithelfalten bei F. diplochaitus (Abb. 1). In den unteren liegt der Pharynxbulbus, der zunächst in eine rechte und linke Hälfte geteilt ist (Abb. $3 \mathrm{~b}-\mathrm{e}$ ). Caudalwärts wird nun die Offnung der Pharynxtasche ventral dadurch verschlossen, daß sich linkes und rechtes Epithel unterhalb der beiden Bulbusteile vereinigen. Das Lumen der Pharynxtasche erhält dadurch die Form zweier senkrecht übereinander und jeweils kopfstehender $T$ (Abb. 3e).

Nun beginnen sich rechte und linke Bulbushälfte zu vereinigen. Dadurch wird die bisher einheitliche Pharynxtasche in zwei Teile gespalten (Abb. 3f), deren Lumina nicht mehr miteinander in Verbindung stehen. Ventral vom nunmehr im Querschnitt 
halbmondförmigen Bulbus liegt ein Teilstück der Pharynxtasche, das kurz darauf blind endigt. Der dorsale Anteil hat nach wie vor Verbindung mit dem Vorderdarmlumen (Abb. 3g).

Schließlich wird aber auch diese Verbindung unterbrochen, so daß nunmehr Darmund Pharynxtaschenlumen voneinander getrennt sind (Abb. 3h-i). Die Pharynxtasche
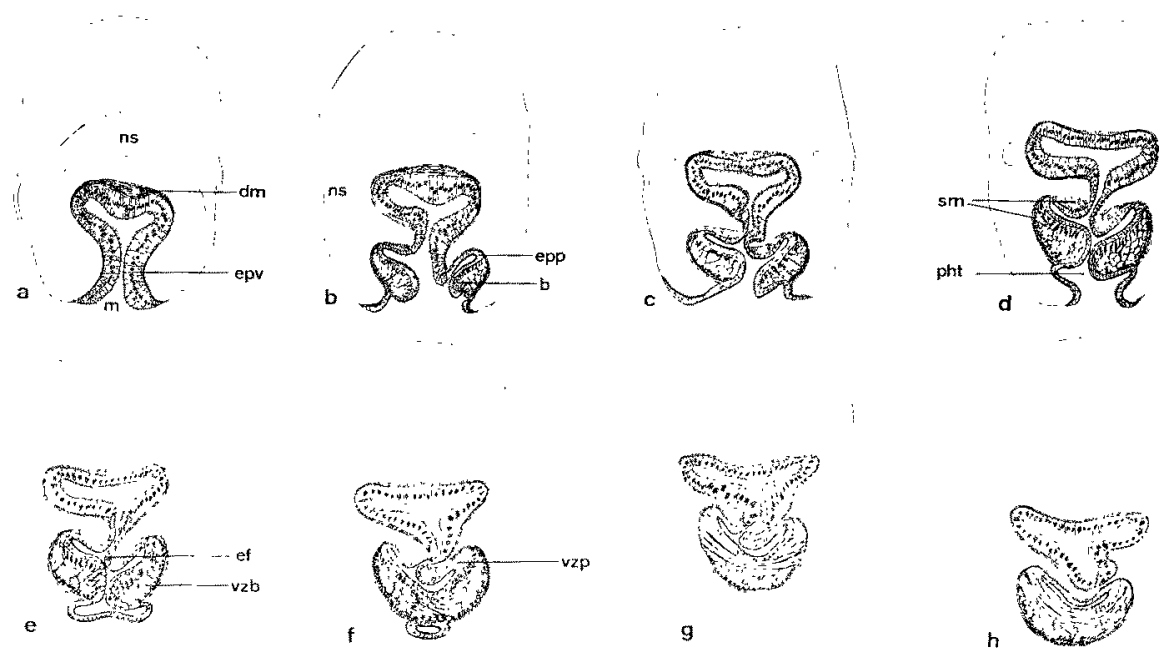

$150 . \mathrm{mm}$
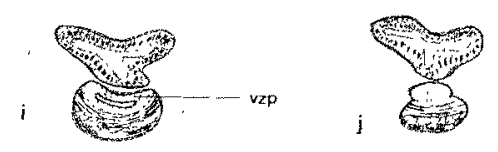

Abb. 3: Schematische Querschnitte $(a-j)$ durch den Vorderkörper von Stylarioides monolifer

nimmt dann die halbmondförmige Form des darunterliegenden Bulbus an. Tasche und Bulbus endigen schließlich blind auf gleicher Höhe (Abb. 3j).

Der Darm ist allseits von einer kräftigen Muskulatur umgeben. Wie bei $F$. diplochaitus ist auch bei $S$. monolifer ein den Bulbus einhüllendes Sagittalmuskelsystem vorhanden (Abb. 3). Es umgreift die beiden Bulbus-Hälften zunächst an den Lateralseiten; nach der Bildung des einheitlichen Bulbus liegt es ihm auch ventral an. Umbiegungsstelle vom ventralen zum dorsalen Anteil der Sagittalmuskel-Fasern ist wieder distales Bulbus- bzw. Pharynxtaschenende. Die dorsalen Muskelteile enden zum größten Teil innerhalb der Epithelfalten ( $\mathrm{Abb} .3 \mathrm{c}-\mathrm{g}$ ), einige auch am ventralen Teil des Vorderdarms. Am distalen Bulbus-Ende setzt ein Bulbus-Retraktor an, der caudalwärts zieht und im ventralen Hautmuskelschlauch aufgeht. 


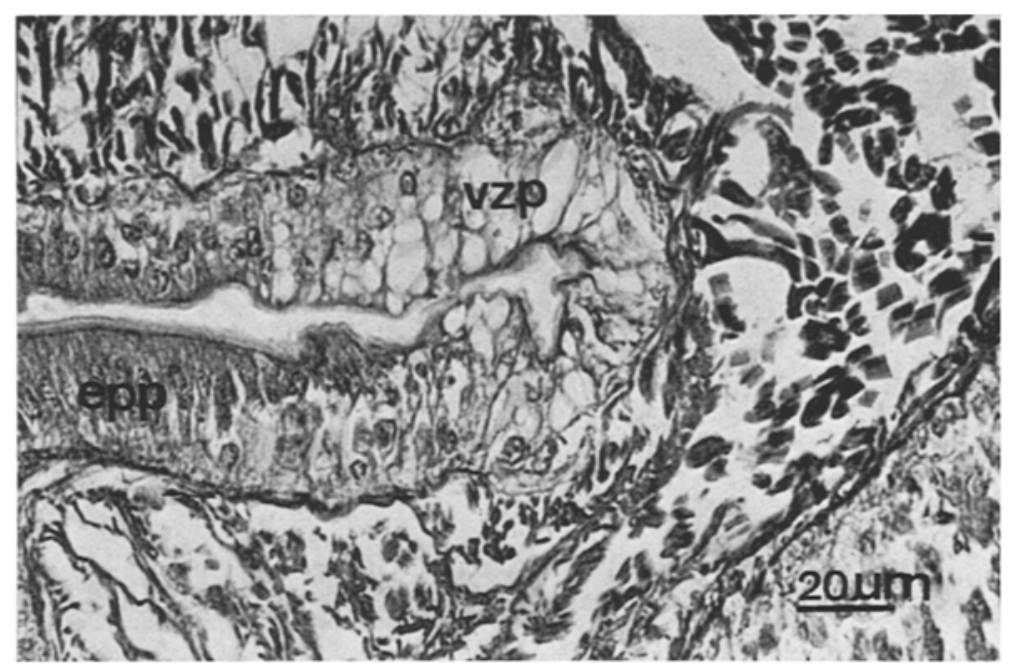

Abb. 4 : Blindgeschlossenes Ende einer lateralen Aussackung der Pharynxtasche von Stylarioides monolifer

Histologie von Vorderdarm, Bulbus und Pharynxtasche bei Stylarioides monolifer

Das Vorderdarmepithel (Abb. 3a-j) besteht aus etwa $40 \mu \mathrm{m}$ großen Zellen, die, bis auf eingestreute Drüsenzellen, mit Cilien versehen sind. Das Epithel der Pharynxtasche dagegen wird aus wesentlich kleineren, $15 \mu \mathrm{m}$ großen Zellen gebildet (Abb. 3, 4). Sie tragen keine Cilien, sondern sind wie bei $F$. diplochaitus von einer Kutikula bedeckt.

Neben diesen zylindrischen Epithelzellen kommt im Pharynxtaschenepithel noch ein zweiter Zelltyp vor. Er beginnt nach der Hälfte der insgesamt ca. $2 \mathrm{~mm}$ langen Pharynxtasche. Es treten hier "Blasenzellen" auf, die denen von $F$. diplochaitus entsprechen (Abb. 3f-j, 4).

Solche Blasenzellen bilden zunächst nur die Auskleidung des blindgeschlossenen Endes der oberen, lateralen Pharynxtaschen-Aussackung (Abb. $3 f-g$ ). In distaler Richtung werden sie dann jedoch immer zahlreicher, bis sie schließlich am Ende der Pharynxtasche als alleiniger Epitheltyp vorhanden sind (Abb. $3 \mathrm{~h}-\mathrm{j}, 5$ ). Die Blasenzellen bilden ebenfalls eine Kutikula aus; das Pharynxtaschenepithel ist somit lückenlos von einer Kutikula ausgekleidet.

Der histologische Aufbau des Pharynxbulbus entspricht dem von $F$. diplochaitus. Zwei Zelltypen kommen vor: langgestreckte Muskelzellen sowie organellarme Blasenzellen (Abb. 3, 5).

Die Blasenzellen erfüllen das Innere des Bulbus, seine Wand wird von Muskelzellen gebildet, die ringförmig die inneren Zellen umschließen. Wie bei $F$. diplochaitus durchqueren einzelne langgestreckte Muskelfasern den Bulbus auch in dorsoventraler Richtung. 


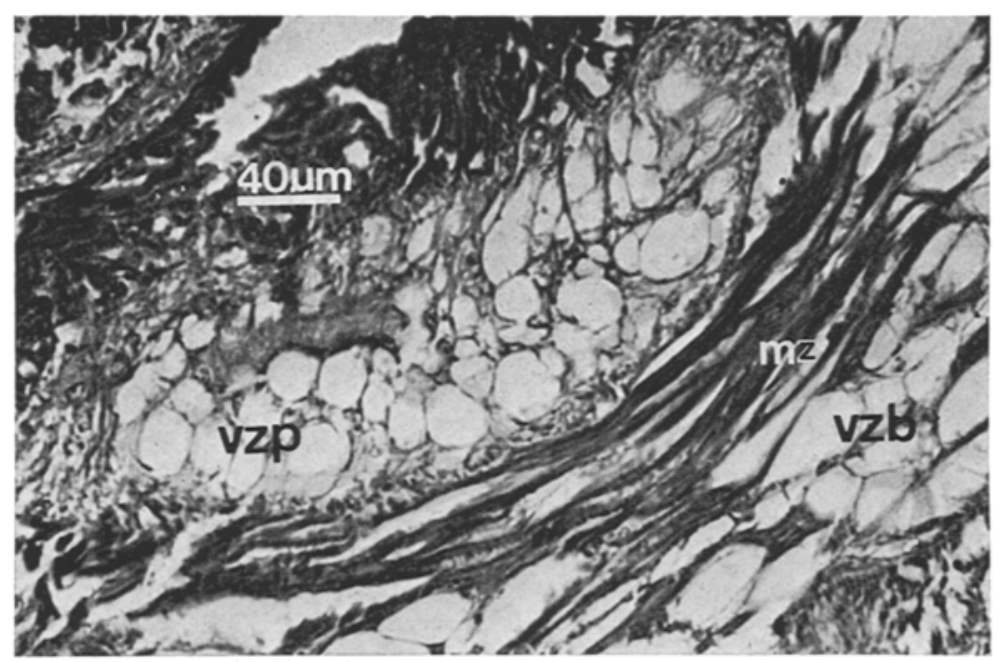

Abb. 5: Querschnitt durch den distalen Teil von Pharynxtasche und Pharynxbulbus von Stylarioides monolifer (die Abbildung entspricht in etwa dem Bild 3j)

\section{DISKUSSION}

Die vorliegenden Ergebnisse zeigen unter Berücksichtigung bzw. Interpretation der kurzen Mitteilungen von Günther (1912), Schumprer (1927), JeEner (1932) und OrRhage (1973), daß den Flabelligeriden ein ventral gelegener Pharynxbulbus zugesprochen werden muß.

Damit ist Dales' Auffassung $(1962,1963)$ vom Fehlen eines solchen Bulbus bei dieser Polychaeten-Familie hinfällig. Die Korrektur dieser Ansicht bestätigt die Warnung Orrhages (1973), daß bisher zu wenige Polychaeten im Hinblick auf die eingangs erwähnte Vorstellung von DALEs untersucht wurden, nach der es möglich ist, Polychaeten auf Grund des Vorhandenseins oder Fehlens eines Pharynxbulbus zu klassifizieren. Erst ein detaillierter Vergleich von Vorkommen, Lage und Bau eines solchen Bulbus kann zeigen, inwieweit sich mit dieser Struktur phylogenetische Aussagen machen lassen.

\section{Baudes Bulbusund der Pharynxtasche bei Flabelligeriden}

Die an den beiden untersuchten Arten gewonnenen Ergebnisse zeigen, daß im Bau des Bulbus und der Pharynxtasche bei beiden Gattungen mehrere grundsätzliche Gemeinsamkeiten bestehen.

Charakteristischerweise ist das Pharynxtaschenepithel von einer Kutikula bedeckt und kann dadurch deutlich vom übrigen Vorderdarmepithel, das Cilien trägt, unterschieden werden. Das Auftreten von Blasenzellen im Pharynxtaschenepithel muß 
als typische Struktur der Flabelligeriden gedeutet werden. Aus den Bildern Orrhages (1973, Abb. 6-9) geht hervor, daß der gleiche Zelltyp an entsprechender Stelle auch bei Brada villosa auftritt.

Insgesamt scheint die Pharynxtasche von Flabelligera diplocbaitus in ihrem Bau der von Brada villosa zu entsprechen (OrRHAGe, 1973), während die von Stylarioides monolifer durch laterale und ventrale Aussackungen komplizierter gestaltet ist. Möglicherweise zeigt in dieser Hinsicht Pherusa plumosa einen ähnlichen Bau, wie sich aus den Abbildungen Schliepers (1927) ergibt.

Für die untersuchten Gattungen sind zwei ins Pharynxtaschen-Lumen springende Falten charakteristisch. Sie dienen als Anheftungsfläche für den Sagittalmuskel, der von dort bogenförmig proximad verläuft und die Ventral- und Lateralseite des Bulbus abschließt. Der Muskel endet jeweils an der ventralen Epithelauskleidung der Pharynxtasche.

Der Bulbusretraktor zwischen Bulbus-Hinterende und Hautmuskelschlauch scheint ebenfalls allgemein bei Flabelligeriden vorzukommen, wie den Angaben bzw. Abbildungen bei SCHLIEPER (1927) und OrRHAGE (1973) zu entnehmen ist.

Der Bulbus selbst ist eine in sich abgeschlossene Struktur. Sein Bau bei S. monolifer ist insofern ungewöhnlich, weil er die Form eines langgestreckten $U$ besitzt, dessen beiden proximalen Abschnitte links und rechts vom Pharynxtaschen-Lumen liegen. Dadurch entsteht der Eindruck eines zunächst paarigen Organs. Bei $F$. diplochaitus ist der Bulbus dagegen von kompakt einheitlichem Bau, nur seine Vorderfläche ist etwas vertieft, so daß proximal zwei kleine Vorsprünge nach vorn ragen. Gleiches dürfte auch für Brada villosa gelten, wie sich nach den Abbildungen OrRHAGEs (1973) rekonstruieren läßt.

Typisch für den Bau des Bulbus sind zirkuläre und dorsoventrale Muskelfasern sowie große Blasenzellen in seinem Innern. Beide Zelltypen gewährleisten die große Elastizität und Steifheit des Bulbus, der hervorstreckbar ist (GüNTHER, 1912; SCHLIEPER, 1927; JEENER, 1932).

\section{Vergleich mit anderen Polychaeten}

Eingehendere Untersuchungen am Bulbus von Anneliden liegen vor für verschiedene Ctenodriliden (Galvagnt, 1903; Wilfert, 1973, 1974), "Archianneliden “ (Pierantoni, 1908; Jaegersten, 1947; Ohm, 1963), Parergodriliden (Karling, 1958; ReiSINGer, 1960), Potamodriliden (Bunke, 1967), Terebelliden (SutTon, 1957) und Ariciiden (EIsIG, 1914).

Danach lassen sich die beschriebenen Organe in folgende Typen einteilen:

(I) Bulbus nicht hervorstreckbar: Potamodrilus.

(II) Bulbus hervorstreckbar.

(1) Muskulöser Pharynxbulbus (aud bei Parergodrilus?) in Verbindung mit einer drüsigen Schlundtasche: Parergodriliden.

(2) In sich abgeschlossener Bulbus, mit Sagittal- und Rückziehmuskel.

(a) Bulbus aus plattenähnlichen Muskelzellen aufgebaut: "Archianneliden" (alle?), „primärer Rüssel“ der Ariciiden (?). 
(b) Bulbus aus Muskel- und Blasenzellen aufgebaut: Ctenodriliden, Terebelliden.

Diese Aufreihung kann sich nur auf die wenigen detaillierten anatomisch-histologischen Untersuchungen beziehen und muß die kurzen Erwähnungen eines Pharynxbulbus innerhalb des Rahmens größerer Polychaeten-Beschreibungen außer acht lassen. Danach würde der Bulbus der Flabelligeriden zur letzten Kategorie zu zählen sein.

Gegenüber den dort genannten Gruppen zeichnen sich die Flabelligeriden durch einen sehr einheitlichen Bau ihres Bulbus aus: die Blasenzellen liegen im Bulbus-Innern, werden durchquert von dorsoventralen Muskelfasern und zirkulär von ihnen umgeben. Bei Apbropharynx heterochaeta z. B. (Familie Ctenodrilidae; WILFERT, 1974) sind Blasen- und Muskelzellen stockwerkartig voneinander getrennt; Ctenodrilus serratus aus der gleichen Familie hat einen davon abweichend konstruierten Bulbus (WILFERT, 1973).

Aus folgenden zwei Hauptgründen ist bis zum jetzigen Zeitpunkt ein genauer Vergleich der einzelnen Bulbus-Typen innerhalb der Polychaeten, besonders im Hinblick auf die Frage von Homologie und Konvergenz dieses Organs noch nicht möglich:

(1) Es muß damit gerechnet werden, daß unter den von Dales $(1962,1963)$ als Pharynxbulbus subsummierten Strukturen sich möglicherweise sehr unterschiedlich gebaute Organe befinden. Eigene Untersuchungen an Owenia fusiforme z. B. zeigten, daß ein Bulbus vorhanden ist, der nur aus Blasenzellen bestcht und daß sich seine Lagebeziehung zu anderen Organen deutlich von dem Bulbus der Flabelligeriden oder der Ctenodriliden (WIIfERT, 1973, 1974) unterscheidet. Ahnliches scheint für die Familie Magelonidae zu gelten. Der ventrale Bulbus von Magelona z. B. scheint nach der Abbildung bei Orrhage (1973) einen Aufbau zu haben, der deutlich von der BulbusKonstruktion anderer Polychaeten-Familien abweicht.

(2) Eigene elektronenmikroskopische Untersuchungen an einigen Polychaeten (Terebellidae, Ampharetidae) ließen erkennen, daß vielfach die lichtmikroskopischen Beschreibungen des Pharynxbulbus nicht völlig zutreffend sind (z. B. Sutton, 1957; Fauver, 1897). Diese Hinweise unterstützen nachdrücklich OrRHages Forderung (1973) nach ausreichender Untersuchung möglichst vieler Polydhaeten im Hinblick auf das Problem "Pharynxapparat".

\section{Der Bulbus von Poeobius meseres und den Flabelligeriden}

Die systematische Stellung des von HeATH (1930) beschriebenen Poeobius meseres ist bis heute umstritten (vgl. RobBrns, 1965). Die meisten Autoren sehen in Poeobius einen Polychaeten, der in vieler Hinsicht Beziehungen $\mathrm{zu}$ den Flabelligeriden aufweist.

Robbins (1965) untersuchte den seit Heath (1930) bekannten Pharynxbulbus von $P$. meseres näher. Sie fand, daß der Bulbus aus zwei Teilen besteht: ein vorderer, mit großen Blasenzellen (Roвbins: „fluid-filled cells") erfüllter Abschnitt wird von einem hinteren, der stark muskulös ist, durch einen Transversalmuskel getrennt. Ein kleiner Bezirk auf der Ventralseite des Poeobius-Bulbus trägt Cilien; dies ist von keinem anderen Polychaeten bekannt. RoBbins (1965) berief sich bei ihrer Feststel- 
lung, daß den Flabelligeriden kein Pharynxbulbus zukäme, auf DALEs (1962). Obwohl diese Angabe nun nicht mehr aufrechterhalten werden kann, kann die Tatsache, daß $P$. meseres und Flabelligeriden einen Bulbus besitzen, nicht als Indiz einer näheren Verwandtschaft herangezogen werden. Der Bulbus von $P$. meseres ist zweigeteilt (wie z. B. auch in anderer Form bei Terebelliden, s. z. B. SutTon, 1957), bei den Flabelligeriden einheitlich. Blasenzellen kommen bei vielen anderen Polychaeten im Bulbus vor; ihr Verteilungsmuster innerhalb des Bulbus stimmt nicht mit dem bei Flabelligeriden überein. Und Muskelzellen sind geradezu das Charakteristikum eines in sich abgeschlossenen Polychaeten-Bulbus.

Selbst wenn die Ubbereinstimmungen größer wären, müßte unter Berücksichtigung der erwähnten Vorbehalte davor gewarnt werden, irgendwelche systematisch-taxonomischen Schlußfolgerungen aus ihnen zu ziehen.

\section{ZUSAMMENFASSUNG}

1. Anatomisch-histologisch wurde der Vorderdarm von Flabelligera diplochaitus und Stylarioides monolifer aus der Polychaeten-Familie Flabelligeridae untersucht. Die Auswertung ergab, daß den Flabelligeriden ein Pharynxbulbus zugesprochen werden muß.

2. Wesentliche Teile des Flabelligeriden-Schlundapparats sind: eine ventral gelegene Pharynxtasche, deren kutikularisiertes Epithel aus einfachen Zylinder- sowie Blasenzellen besteht, ein Bulbus aus Muskel- und Blasenzellen, ein Sagittalmuskel und ein Retraktor des Bulbus.

3. Ein Vergleich mit den Schlundapparaten anderer Anneliden zeigt, daß vor allem innerhalb der Polychaeten noch zuwenige detaillierte Untersuchungen vorliegen, die es gestatten, vom Bau des Pharynxbulbus aus phylogenetische Schlußfolgerungen zu ziehen.

\section{ZITIERTE LITERATUR}

Bunke, D., 1967. Zur Morphologie und Systematik der Aeolosomatidae Beddard 1895 und Potamodrilidae nov. fam. (Oligochaeta). Zool. Jb. (Syst. Okol. Geogr. Tiere) 94, 187 bis 368 .

DALES, R. P., 1962. The polychaete stomodeum and the inter-relationships of the families of Polychaeta. Proc. zool. Soc. Lond. 139, 389-428.

- 1963. Annelids. Hutchinson, London, 200 pp.

EIsıG, H., 1914. Zur Systematik, Anatomie und Morphologie der Ariciiden nebst Beiträgen zur generellen Systematik. Mitt. zool. Stn Neapel 21, 153-593.

Fauves, P., 1897. Recherches sur les ampharétiens, annélides polychètes sédentaires, morphologie, anatomie, histologie, physiologie. Danel, Lille, 212 pp.

Galvagni, E., 1903. Histologie des Genus Ctenodrilus Clap. Arb. zool. Inst. Univ. Wien 15, 46-78.

GüNTHER, K., 1912. Beiträge zur Systematik der Gattung Flabelligera und Studien über den Bau von Flabelligera (Siphonostoma) diplochaitus OTTo. Jena Z. Naturwiss. 48, 93-186.

HeAth, H., 1930. A connecting link between the Annelida and the Echiuroidea (Gephyrea armata). J. Morph. 49, 223-249. 
Jaegersten, G., 1947. On the structure of the pharynx of the Archiannelida with special reference to there-occurring muscle cells of aberrant type. Zool. Bidr. Upps. 25, 551-570. JEENER, R., 1932. Evolution morphologique et fonctionelle du stomodeum des polychètes. Recl Inst. zool. Torley-Rousseau 4, 5-30.

Karding, T. G., 1958. Zur Kenntnis von Stygocapitella subterranea Knöldner und Parergodrilus heideri ReIsINGER (Annelida). Ark. Zool. 11, 307-342.

Oнм, G., 1963. Uber den Bau des Pharynx einiger Archianneliden. Zool. Anz. 171, 179-203.

OrRHAGe, L., 1964. Anatomische und morphologische Studien über die Polychaetenfamilien Spionidae, Disomidae und Poecilochaetidae. Zool. Bidr. Upps. 36, 335-405.

-- 1973. Two fundamental requirements for phylogenetic-scientific works as a background for an analysis of DALEs's and WeBB's theories. Z. zool. Syst. Evolutionsforsch. 11, 161-173.

- 1974. Uber die Anatomie, Histologie und Verwandtschaft der Apistobranchidae (Polychaeta Sedentaria) nebst Bemerkungen über die systematische Stellung der Archianneliden. Z. Morph. Tiere 79, 1-45.

Pierantoni, U., 1908. Protodrilus. Fauna Flora Golf. Neapel 31, 1-226.

Reisinger, E., 1960. Die Lösung des Parergodrilus-Problems. Z. Morph. Okol. Tiere 48, 517544.

RoBrins, D. E., 1965. The biology and morphology of the pelagic annelid Poeobizs meseres HEATH. J. Zool. 146, 197-212.

SChLIEPER, C., 1927. Stylarioides plumosus, eine monographische Darstellung. Z. Morph. Okol. Tiere 7, 320-383.

Storch, V., 1968. Zur vergleichenden Anatomie der segmentalen Muskelsysteme und zur Verwandtschaft der Polychaeten-Familicn. Z. Morph. Tiere 63, 251-432.

Sutton, M. F. 1957. The feeding mechanism, funcrional morphology and histology of the alimentary canal of Terebella lapidaria L. (Polychacta). Proc. zool. Soc. Lond. 129, 487-523.

Welsch, U. \& Storch, V., 1972. Einführung in Cytologie und Histologie der Tiere. Fischer, Stuttgart, 244 pp.

WyLffrT, M., 1973. Ein Beitrag zur Morphologie, Biologie und systematischen Stcllung des Polychaeten Ctenodrilus serratus. Helgoländer wiss. Meeresunters. 25, 332--346.

- 1974. Aphropharynx beterochaeta nov. gen. nov. spec., ein neuer Polychaet aus der Familie Ctenodrilidae KenNel 1882. Cah. Biol, mar. 15, 495-504.

Anschrift des Verfassers: Dr. M. WILFERT

Institut für Zoologic

D-4 Düsseldorf 1

Mettmanner Str. $16 / 18$

Bundesrepublik Deutschland 